\title{
Компьютерная программа
}

\section{когнитивно-поведенческой терапии для мобильных приложений} «АНТИ-ДЕПРЕССИЯ»:

\section{результаты исследования эффективности}

Трочикая О.В., к.пол.н., директор іCоgnito, преподаватель МГИМО Батхина А.А., к.псих.н., научный сотрудник НИУ ВШЭ

Абстракт: В статье приводятся исследования результативности программы «Анти-депрессия» iCognito ${ }^{1}$, разработанной на основе методов когнитивно-поведенческой терапии для массового использования. Программа реализуется в формате микро-сессий с чатботом в мобильном приложении. Исследование подтвердило, что через две недели пользователи программы «Анти-депрессия» продемонстрировали снижение уровня депрессии, тревожности, стресса, руминаций и проблем со сном; повышение уровня сострадания к себе, осознанности, позитивной ориентации на решение проблем, самоэффективности, субъективного восприятия благополучия и оптимизма.

\section{Введение}

Депрессия - широко распространенное психическое расстройство, которое эффективно лечится психотерапевтическими и фармакологическими методами. Однако, по оценкам ВОЗ, до 85\%

\footnotetext{
${ }^{1}$ Исключительное право на Программу принадлежит Обществу с ограниченной ответственностью «АЙКОГНИТО». Программа зарегистрирована в Федеральной службе по интеллектуальной собственности, патентам и товарным знакам. Свидетельство об официальной регистрации программы №2020665010 зарегистрировано в реестре программ для ЭВМ от 22.11.2020 г. Программу можно бесплатно загрузить на смартфон или планшет из Google Play Store по ссылке: https:/play.google.com/store/apps/details?id=com.icognito.depressionж; из Apple App Store по ссылке: https://apps.apple.com/ru/app/aнти-депрессия/id1528842077
} 
населения в странах с низким и средним уровнем доходов не получают лечения из-за нехватки ресурсов, отсутствия квалифицированных поставщиков медицинских услуг и социальной стигмы, связанной с проблемами психического здоровья (ВО3, 2021 г.). Решение проблемы связывается с распространением информационных технологий. Электронные инструменты диагностики, мониторинга, психообразования и терапии являются «неисчерпаемыми» ресурсами и могут сыграть важную роль там, где человеческих ресурсов недостаточно (Munoz, 2010).

Опыт развитых стран показывает, что программы компьютерной когнитивно-поведенческой терапии (ККПТ), такие как Beating the blues (Великобритания), Deprexis (Германия), Good Days Ahead (США) и Mood Gym (Австралия) представляют собой удобное и экономичное решение для расширения доступа к психиатрической помощи. Хотя эффект ККПТ ниже по сравнению с индивидуальной терапией, программы приводят к значительному снижению уровня депрессии и способны оказывать качественную психологическую помощь в массовом масштабе - в сочетании с консультациями специалистов или в качестве самостоятельных инструментов самопомощи (Wright 2019; 2020; Carlbring 2018; Thase 2018; Berger 2018; Andrews 2010; Richards 2012; Foroushani 2011; So 2013).

Важными цифровыми инструментами психиатрической помощи становятся мобильные приложения для смартфонов. Несмотря на то, что 
мобильные приложения имеют ограниченную функциональность по сравнению с веб-программами, они все чаще используются в качестве карманных помощников для проигрывания медитаций, записи мыслей, мониторинга настроения, планирования действий, своевременного приема таблеток и проведения проверочных тестов (Caldeira 2017; Stawartz 2018). Хотя многие приложения вызывают опасения по поводу качества контента и проблем с конфиденциальностью (Marshall 2019; Marshall 2020), было собрано достаточно доказательств того, что профессионально разработанные приложения могут давать небольшой или умеренный положительный эффект при постоянном использовании (Huguet 2016; Firth et al.2017).

\section{Ситуация в России}

Программа «Антидепрессия» iCognito - это первая компьютерная программа на основе когнитивно-поведенческой терапии на русском языке, разработанная профессиональными психотерапевтами и прошедшая научные исследования эффективности. «Анти-депрессия» была создана, чтобы расширить доступ российских граждан к базовой психологической помощи и восполнить пробел в недостаточном уровне оказания психиатрической помощи в России.

Распространенность депрессии в России сопоставима с уровнем европейских стран и составляет от 6 до 10\% в зависимости от города (Torre 2021; Cook 2020). Однако по уровню лечения Россия значительно 
отстает от развитых стран. Хотя уровень смертности от самоубийств снизился с 54 смертей на 100 тыс.чел. в 2000 году до 25 в 2018 году, он попрежнему в 2,5 раза выше среднего уровня стран Европейского Союза, который составляет 11 смертей на 100 тыс.чел. (ВОЗ 2021).

Согласно недавнему исследованию, проведенному в двух городах России, большинство лиц, страдающих от депрессии, не имеют подтвержденного диагноза, так как никогда не обращались с этими симптомами к специалистам. Менее $1 \%$ мужчин и $4 \%$ женщин в России с диагнозом умеренная депрессия использовали антидепрессанты (Cook et al., 2020). Это контрастирует со статистикой в Англии, где 70\% людей с симптомами депрессии получили диагноз депрессии в разные периоды своей жизни или со статистикой США, где 27\% населения, у которых было диагностировано умеренная депрессия, получали фармакологическое лечение (McManus, 2016).

Государственные психиатрические учреждения в России традиционно уделяют особое внимание лечению тяжелых неврологических заболеваний, усиливая социальную стигму и опасения получить ярлык «пациента психиатрической больницы» (Jenkins et al., 2007; Краснов и Гурович, 2012; Winkler et al., 2017). Социальные службы по всей стране предоставляют бесплатные психологические консультации очно, по телефону или через Интернет, но помощь ограничена по времени и объему. Разговорная терапия доступна только в частных учреждениях по 
относительно высокой цене (стоимость курса из 10 консультаций равна 35-40 тыс.рублей, что сопоставимо с месячным доходом среднего жителя России).

В результате в 2018 году только 2\% россиян сообщили, что когдалибо посещали психолога или психотерапевта. Доля тех, кто отвергает идею обращения за профессиональной помощью в тяжелые времена, увеличилась с 47\% в 2006 г. до $71 \%$ в 2018 г. Среди причин отказа респонденты называют недоверие к профессии психолога и высокую стоимость услуг (см. опрос Фонда общественного мнения, 2020 г.). Население в России в целом мало осведомлено о проблемах психического здоровья и о природе депрессивного расстройства в частности: по сравнению с участниками из США, российские участники одного исследования с большей вероятностью рассматривали депрессию как проявление слабой воли или аморального образа жизни, и полагали, что человек должен самостоятельно бороться с расстройствами настроения (Нерсессова, 2019).

\section{Описание программы «Анти-депрессия» iCognito}

Целью разработчиков было создать универсальный инструмент самопомощи, доступный массовой аудитории и не требующий специализированных компьютерных навыков и даже наличия компьютера. Программа iCognito «Антидепрессия» была разработана как кросс- 
платформенное мобильное приложение, доступное для использования на смартфонах и планшетах операционных систем Android и iOS.

Выбор формата обуславливается тем, что распространенность мобильных телефонов в России и в мире уже превысила распространенность персональных компьютеров, а также тем, что смартфоны, как правило, всегда у человека под рукой и более удобны для регулярных коротких занятий.

Курс «Анти-депрессия» может использоваться пользователем самостоятельно или в сочетании с консультациями психотерапевта, подписку на которые можно оформить в самом приложении. Приложение «Анти-депрессия» можно бесплатно загрузить из Google Play Store и Apple App Store. После установки приложения каждый пользователь должен пройти регистрацию и создать учетную запись (ввести имя, почту и пароль).

Программа «Анти-депрессия» содержит:

- Курс занятий в формате диалогов с виртуальным психологом Максом. Все занятия разбиты на 7 тематических модулей по 46 микро-сессий длительностью 7-15 минут.

- Раздел «Техники», в которых есть дневник мыслей, дневник благодарности и медитации осознанности. 
- Раздел «Чат с психологом», в котором пользователь может общаться со специалистом при оформлении платной подписки.

Клиент может пройти программу в любом темпе. Рекомендуемый срок прохождения - от 1 месяца (при самостоятельном освоении) до 2 месяцев (в сочетании с консультациями специалиста).

Программа была разработана в формате чатбота - разговорного агента, имитирующего диалог с пользователем в окне для обмена текстовыми сообщениями по определенному алгоритму. Формат чатбота имеет несколько преимуществ перед другими форматами онлайнпрограмм:

- Разговорный стиль понятен и доступен людям разного возраста, уровня образования и профессии,

- Чатбот поддерживает высокую вовлеченность за счет интерактивности: пользователь должен совершать действие в среднем 3-4 раза в минуту - выбрать готовый ответ из предложенных или записывать свой вариант ответа,

- чатбот обеспечивает постоянное внимание пользователя на занятиях: чтобы увидеть продолжение диалога, пользователь обязан отвечать на вопросы. У пользователя нет возможности отвлечься, пропустить информацию или слушать в пассивном 
режиме, что обеспечивает высокую результативность формата в сжатые сроки.

Программа состоит из 7 модулей:

Модуль 1 «Диагностика» содержит: 1) тесты на депрессивное и тревожное расстройство по опросникам PHQ-9 и GAD-7; 2) обучающее занятие на тему «Что такое депрессия и почему важно ее лечить», 3) упражнение на постановку целей (McCarter 2008; Arkowitz 2008).

Модуль 2 «Поведенческая активация» содержит: 1) обучающее занятие о том, что такое поведенческая активация и как важно позитивное подкрепление после каждого выполненного действия, 2) практическое занятие на планирование практических дел, 3) практическое занятие на планирование приятных дел (Kanter 2009). В последнем занятии модуля виртуальный психолог предлагает составить список личных ценностей и обсудить, как действия, направленные на достижение ценности, могут сделать жизнь более значимой и осмысленной (Kirschenbaum 2013).

Модуль 3 «Когнитивное перестроение» содержит: 1) обучающее занятие о принципах когнитивно-поведенческой терапии, объясняющее, как негативное мышление влияет на наше настроение, и почему негативным мыслям не всегда нужно верить; 2) практические занятия на распознавание и исправление ошибок «чрезмерного обобщения», «навешивание ярлыков», ошибки «катастрофизации». В заключительной сессии модуля о руминации клиент узнает, что такое бесплодные 
размышления, почему руминации контрпродуктивны в решении проблем и способствуют поддержанию депрессии (Beck 1987; Burns 2012; Watkins 2016)

Модуль 4 «Осознанность» содержит: 1) обучающее занятие на тему, что такое майндфулнесс, почему важно обращать внимание на собственные чувства, сосредотачиваться на настоящем моменте и понимать связь между событиями и эмоциями; 2) практические занятия, объясняющие последовательность действий при выполнении практик осознанности - глубокого дыхания, нервно-мышечной релаксации, визуализации, переключения и др.(Segal 2012)

Модуль 5 «Сострадание» работает с самообвинением и негативным отношением к себе. Модуль содержит: 1) обучающее занятие на тему того, что такое сострадательное отношение к себе и почему самокритика не помогает «исправиться», а только ухудшает ситуацию, 2) практические занятия, как выражать поддержку себе и другим, как заботиться о себе. (Guilbert 2018)

Модуль 6 «Решение проблем» содержит: 1) обучающее занятие на тему позитивной и негативной проблемой ориентации, тестирование клиента для выяснения его собственной проблемной ориентации, 2) обучающее занятие на тему универсального алгоритма решения проблем, 3) практическое занятие по применению алгоритма к решению собственных проблем (Mezu 2012) 
Модуль 7 «Эффективное общение» содержит: 1) обучающее занятие о правилах эффективной коммуникации, 2) обучающее занятие о правилах активного слушания, 3) практическое занятие по формулированию эффективного высказывания (Я-сообщения), 4) практическое занятие по разрешению конфликтов (Markowitz 2004).

Для тренировки освоенных знаний и навыков пользователям рекомендуется заполнять дневники мыслей и дневник благодарности в электронных формах в мобильном приложении, а также прослушивать аудио-гиды по практикам осознанности - глубокого дыхания, нервномышечной релаксации, переключения внимания, визуализации и др. (также содержатся в мобильном приложении в разделе «Техники»).

\section{Метод}

В настоящем исследовании оценивалась эффективность программы «Анти-депрессия» iCognito по сравнению с контрольной группой (лист ожидания). Мы ожидали как внутригрупповые изменения в экспериментальной группе, так и межгрупповые различия с контрольным условиям. Были предложены следующие исследовательские гипотезы.

H1. Программа «Анти-депрессия» iCognito уменьшает симптомы депрессии, беспокойства и стресса. 
Н2. Программа «Анти-депрессия» iCognito развивает сострадание к себе, внимательность, позитивную ориентацию на решение проблем, самоэффективность, субъективное благополучие и оптимизм.

H3. Программа «Анти-депрессия» iCognito снижает уровень руминации, негативную ориентацию на решение проблем и выраженность проблем со сном.

\section{Выборка}

Общая выборка исследования составила $\mathrm{N}=73$ (70 женщин и 3 мужчин) в возрасте от 18 до 57 лет $(\mathrm{M}=28,13 ; \mathrm{SD}=7,48)$. Первую анкету заполнили 149 человек. Таким образом, наша выборка была преимущественно женской. Второй опросник заполнили 35 из 101 (34,65\%) участника экспериментальной группы и 38 из 48 (79,16\%) участников контрольной группы. Более подробная информация о социально-демографических характеристиках выборки представлена в Таблице 1.

Хотя высокий уровень отсева снижает достоверность результатов, он типичен для исследований в области e-health и не обязательно отражает программные недостатки (Eysenbach, 2005). Недавний метааналитический обзор 70 рандомизированных контролируемых испытаний вмешательств с использованием смартфонов продемонстрировал, что высокий процент отсева и низкая приверженность исследованию являются обычной 
практикой и типичной особенностью использования мобильных приложений по сравнению с очным посещением специалистов (Linardon et al, 2020).

\section{Прощедура}

Потенциальные участники набирались с помощью рекламы в Facebook co всей России. Рекламные объявления содержали информацию о возможности и условиях участия в исследовательском проекте. Участники, которые переходили по ссылке в объявлении, заполняли список предварительной регистрации с демографическими данными и PHQ-2.

Критериями включения были возраст участников старше 18 лет, наличие не менее 3 из 8 баллов по PHQ-2 и отсутствие на данный момент медицинской или психологической помощи. Мы случайным образом приглашали участников из формы предварительной регистрации в экспериментальную группу или в лист ожидания. Участники экспериментальной группы получили ссылку для установки программы «Анти-депрессия» iCognito и инструкции по ежедневному использованию приложения. В течение двух недель участникам периодически напоминали об использовании приложения. Все участники программы «Анти-депрессия» iCognito имели доступ к технической поддержке во время исследования. Участники из листа ожидания были 
проинформированы о том, что через две недели они получат бесплатный доступ к программе iCognito Анти-Депрессия.

Примерно через две недели участников снова попросили заполнить анкету. Критерием включения в повторный опрос для экспериментальной группы было завершение не менее двух третей программы «Антидепрессия» iCognito; прогресс проверялся в панели администрирования на основании индивидуальных идентификационных кодов, которые участники указывали как при опросе, так и при регистрации в приложении. Участникам было предложено вознаграждение в размере 100 рублей за заполнение первой анкеты и 400 рублей за заполнение второй.

\section{Методики}

Шкала воспринимаемого стресса (PSS) использовалась для оценки уровня стресса (Cohen, Kamarck, \& Mermelstein, 1983). Она состоит из 10 вопросов о прошлом месяце, таких как «За последний месяц, как часто вы расстраивались из-за чего-то, что происходило неожиданно?». Респонденты отвечают по пятибалльной шкале $(0=$ никогда; $5=$ очень часто). Общий показатель суммируется $(\alpha=0,92)$.

Опросник генерализированного тревожного расстройства (GAD-7) использовался для скрининга тревожных симптомов (Spitzer et al., 2006). Он включает 7 вопросов о последних двух неделях, таких как «Чувство нервозности, беспокойства или возбуждения». Респонденты отвечают по 
4-балльной шкале $(1=$ нет; 4 = почти каждый день). Общий показатель суммируется $(\alpha=0,84)$.

Опросник здоровья пациента (PHQ-9) использовался для оценки депрессивных симптомов, он состоит из 9 вопросов о последних двух неделях респондента, таких как «Чувство усталости или недостаток энергии?». Респонденты отвечают по 4-балльной шкале $(1=$ нет; 4 = почти каждый день). Общий показатель суммируется $(\alpha=0,88)$.

Шкала самосострадания Неффа (короткая форма) использовалась для оценки самосострадания (Raes et al., 2010). Шкала состоит из 10 утверждений, таких как «Я стараюсь быть понимающим и терпеливым по отношению к тем аспектам моей личности, которые мне не нравятся». Респонденты отвечают, используя 5-балльную шкалу $(1=$ никогда; 5 = всегда), и общий показатель суммируется $(\alpha=0,78)$.

Шкала руминации (короткая версия) оценивала две когнитивные ориентации - на руминацию (непродуктивный стиль) и рефлексию (продуктивный стиль) (Treynor et al., 2003). Шкала состоит из 11 вопросов, содержащих такие утверждения, как «подумай, а почему я не могу идти?». Респонденты отвечают, используя 4-балльную шкалу (1 = почти никогда; 4 = почти всегда), вычисляются два показателя, соответствующие субшкалам руминации $(\alpha=0,72)$ и рефлексии $(\alpha=0,74)$. 
Опросник нарушения сна PROMIS (короткая форма) использовался для выявления проблем со сном (Yu et al., 2012). Респондент оценивал свой сон за последние 7 дней. Шкала состоит из 8 утверждений таких, как «Я чувствовал раздражение из-за плохого сна». Респонденты отвечают, используя 5-балльную шкалу ( 1 = совсем нет; 5 = очень много). Значения суммируются $(\alpha=0,69)$

Пересмотренный опросник решений сочиальных проблем (D’Zurilla et al., 2002) использовался для оценки положительной проблемной ориентации $(\alpha=0,76)$ и отрицательной ориентации на проблему $(\alpha=0,82)$. Респондент оценивает 11 утверждений, таких как «Я трачу слишком много времени на беспокойство о своих проблемах, вместо того, чтобы пытаться их решить» по 4-балльной шкале ( 1 = совсем не верно; 4 = точно верно), значения суммировались.

Общая шкала самоэффективности была использована для оценки позитивных убеждений о себе (Jerusalem \& Schwarzer, 1981). Респондент оценивает 10 утверждений, таких как «Я всегда смогу решить сложные проблемы, если буду достаточно стараться», используя 4-балльную шкалу $(1=$ совсем не верно; 4 = точно верно). Значения суммируются $(\alpha=.91)$

Шкала субъективного благополучия оценивала благополучие (Diener, 1984). Шкала состоит из 5 вопросов, содержащих такие утверждения, как «В большинстве случаев моя жизнь близка к моему 
идеалу». Респонденты отвечают, используя 5-балльную шкалу $(1=$ категорически не согласен; 5 = полностью согласен), а общий показатель рассчитывается как среднее $(\alpha=0,84)$.

Шкала оптимизма оценивает оптимистическую ориентацию (Pedrosa et al., 2015). Шкала состоит из 9 вопросов, содержащих такие утверждения, как «Я думаю о будущем с уверенностью». Респонденты отвечают, используя 5-балльную шкалу ( 1 = категорически не согласен; 5 $=$ полностью согласен), и общий показатель суммируется $(\alpha=0,74)$.

При повторном измерении респонденты в экспериментальной группе - те, кто использовал программу «Анти-депрессия» iCognito также заполнили анкету пользовательского опыта, в которой оценивались различные аспекты программы (дизайн, удобство использования, технические характеристики) и удобство взаимодействия с чат-ботом.

Исследование также требовало от участников предоставления социально-демографической информации, респонденты указывали свой пол, возраст, уровень образования, уровень дохода, статус отношений, количество детей и прошлый опыт психологической и медицинской поддержки.

\section{Статистический анализ}

Анализ повторных измерений (MANOVA, ANOVA) в IBM SPSS Statistics (версия 24) использовался для анализа эффектов времени, эффектов группы и взаимодействия между временем и группами (эффект 
вмешательства) в точках измерения (Т1 и Т2). Достоверность различий между показателями каждой группы для первого и второго измерений оценивалась с помощью t-критерия Стьюдента для связанных выборок с бутстрэппингом $\mathrm{n}=1000$. Величина эффекта для внутригрупповых различий рассчитывалась с использованием d Коэна. Эквивалентность групп во время первого измерения анализировали с использованием tкритерия Стьюдента для независимых выборок, критерия $\chi 2$ и критерия Манна-Уитни.

\section{Результаты}

\section{Групповая эквивалентность}

В таблице 2 представлена описательная статистика по группам в соответствии с двумя измерениями. Поскольку распределения всех зависимых переменных по данным обоих измерений были довольно близки к нормальному - то есть ни асимметрия, ни эксцесс распределений не превышали 1 по абсолютной величине - использовались параметрические методы для анализа данных. При сравнении баллов участников экспериментальной и контрольной групп по данным второй анкеты (n $=35$ и $\mathrm{n}=38)$ не было различий между группами по социальнодемографическим или психологическим характеристикам, за исключением достоверных различий по возрасту $(\mathrm{U}=94,5, \mathrm{p}=0,012)$ и числу детей $(2=$ $6,043, \mathrm{p}=0,034)$. Таким образом, экспериментальную и контрольную 
группы можно считать равнозначными. Мы могли бы описать нашу выборку как состоящую из женщин, демонстрирующих от умеренного до сильного депрессивного состояния.

\section{Показатель отсева}

Общий показатель отсева составил 51,0\%, причем этот показатель был выше в контрольной группе (20,84\%), чем в экспериментальной (65,35\%). Чтобы изучить переменные, связанные с успешным завершением программы «Анти-депрессия» iCognito, мы сравнили данные из первых анкет участников исследования, которые завершили программу, с анкетами тех, кто начал программу, но остановился до завершения исследования и, следовательно, не сделал этого. По сравнению с теми, кто завершил все исследование, участники экспериментальной группы, которые впоследствии выбыли из исследования и не заполнили вторую анкету, продемонстрировали более низкий уровень самоэффективности и позитивной ориентации на решение проблем. Внутри контрольной группы не было статистически значимых различий по измеряемым показателям между респондентами, заполнившими обе анкеты, и теми, кто заполнил только первую.

\section{Анализ основных эффектов}

Размер выборки (N> 39) достаточен для мощности $=0,80, \alpha=0,05$, размера эффекта как среднего $(f=0,25)$ (рассчитано $\mathrm{G} *$ Power). Последующие результаты основаны на данных респондентов, 
заполнивших обе анкеты $(\mathrm{N}=73)$. В таблице 2 представлены показатели, основанные на первом и втором опросах как для экспериментальной, так и для контрольной группы.

Эффекты программы iCognito Анти-Депрессия оценивались с использованием трех типов анализа. Во-первых, мы оценили значимость различий между первым и вторым измерениями для каждой группы, используя t-критерий Стьюдента для связанных выборок, и величину эффекта, используя d Коэна. Повторные измерения MANOVA и последующие повторные измерения ANOVA также были проведены, чтобы оценить значимость вмешательства, основанного на взаимодействии времени и групповых факторов. В таблице 3 представлены результаты анализа эффектов.

Согласно сравнению измерений, полученных из двух анкет, для экспериментальной группы было получено снижение уровня стресса $(\mathrm{d}=$ 0,854), тревоги $(\mathrm{d}=1,093)$ и депрессии $(\mathrm{d}=1,126)$. Значительно увеличились значения показателей самосострадания $(\mathrm{d}=0,828)$, внимательности $(\mathrm{d}=0,455)$, позитивной ориентации на решение проблем $(\mathrm{d}=0,439)$, самоэффективности $(\mathrm{d}=0,638)$, субъективного благополучия ( $\mathrm{d}=0,447)$ и оптимизма $(\mathrm{d}=0,691)$. В то же время снизились уровни руминации $(\mathrm{d}=1,002)$, нарушения сна $(\mathrm{d}=0,947)$ и негативной ориентации на решение проблем $(\mathrm{d}=0,467)$.

В контрольной группе наблюдались значительные сдвиги в 
отношении стресса $(\mathrm{d}=0,484)$, тревоги $(\mathrm{d}=0,392)$ и депрессии $(\mathrm{d}=0,351)$.

В соответствии с Cramer \& Bock (1966), анализ MANOVA был выполнен в качестве средства защиты от завышения частоты ошибок 1-го типа в последующих ANOVA. Значение Box M 386,54 было связано со значением p 0,008, которое было интерпретировано как несущественное на основании рекомендаций (Huberty \& Petoskey, 2000) (т.e. p <0,005). Таким образом, ковариационные матрицы между группами считались равными для целей MANOVA.

Двусторонний многомерный дисперсионный анализ (MANOVA) был проведен для тестирования модели со всеми зависимыми переменными. Взаимодействие факторов времени и группы получилось значимым [Лямбда Уилкса $=0,194, \mathrm{~F}(12,60)=20,822, \mathrm{p}<0,001, \eta 2=$ 0,806], так же, как временной эффект [Лямбда Уилкса $=0,188, \mathrm{~F}(12,60))$ $=21,566, \mathrm{p}<0,001, \eta 2=0,812]$ и групповой эффект [Лямбда Уилкса $=$ $0,386, \mathrm{~F}(12,60)=7,950, \mathrm{p}<0,001, \eta 2=0,614]$

Перед проведением серии последующих анализов ANOVA предположение об однородности дисперсии было проверено для всех девяти подшкал. На основании серии F-тестов Ливеня предположение об однородности дисперсии было сочтено выполненным. Серия двусторонних ANOVA по каждой из девяти зависимых переменных была проведена в качестве последующих тестов после MANOVA. Как видно из таблицы 3, почти все взаимодействия время х группа были статистически 
значимыми, с величиной эффекта от среднего до высокого.

Результаты показали, что завершение программы «Анти-депрессия» iCognito было связано со снижением стресса $[\mathrm{F}=5,025, \mathrm{p}=0,028,2=$ 0,066], тревожности $[\mathrm{F}=10,272, \mathrm{p}=0,002,2=0,126]$, депрессии $[\mathrm{F}=$ $15,072, \mathrm{p}=0,001,2=0,175]$, руминации $[\mathrm{F}=4,545, \mathrm{p}=0,036,2=0,060]$ и нарушения сна $[\mathrm{F}=14,605, \mathrm{p}=0,001,2=0,171]$, а также повышением уровня сострадания к себе [F $=188,981, \mathrm{p}<0,001,2=0,727]$, осознанности $[\mathrm{F}=6,499, \mathrm{p}=0,013,2=0,084]$, позитивной ориентации на решение проблем [ $\mathrm{F}=8,504, \mathrm{p}=0,005,2=0,107]$, самоэффективности $[\mathrm{F}=4,014, \mathrm{p}$ $=0,049,2=0,054]$, субъективного благополучия $[\mathrm{F}=18,048, \mathrm{p}=0,001,2=$ .203] и оптимизма $[\mathrm{F}=3,883, \mathrm{p}=0,045,2=0,052]$. Однако эффект взаимодействия был незначительным для рефлексии и негативной ориентации на решение проблем. Мы используем предложения Коэна (1988) для интерпретации величины эффектов (малых $(\eta 2=0,01)$, средних $(\eta 2=0,06)$ и больших $(\eta 2=0,14)$ эффектов).

\section{Пользовательский опыт}

Участники экспериментальной группы при повторном измерении дали оценку программе iCognito Анти-Депрессия по 5-балльной шкале. В целом программа была высоко оценена пользователями с точки зрения содержания $(\mathrm{M}=4,27 ; \mathrm{SD}=0,82)$, дизайна $(\mathrm{M}=4,74 ; \mathrm{SD}=0,56)$, простоты использования $(\mathrm{M}=4,63, \mathrm{SD}=0,77)$ и техник $(\mathrm{M}=4,57, \mathrm{SD}=0,79)$. Также участники высоко оценили удобство взаимодействия с чат-ботом (M = 
$4,06, \mathrm{SD}=0,92)$.

\section{Обсуждение}

В проведенном исследовании оценивалась эффективность программы «Анти-депрессия» iCognito по сравнению с контрольной группой (лист ожидания). Исследование подтвердило, что через две недели пользователи программы iCognito Анти-Депрессия продемонстрировали снижение стресса, тревожности, депрессии, руминации и нарушений сна, а также повышение уровня самосострадания, внимательности, позитивной ориентации на решение проблем, осознанности, самоэффективности, субъективного благополучия и оптимизма. Таким образом, наши гипотезы частично подтвердились.

Полученные результаты в целом совпадают с результатами, полученными ранее относительно компьютеризированных программ самопомощи на основе СВТ для лечения депрессии и подтверждающими их эффективность (Andersson et al., 2016; Baumeister et al., 2014; Richardson \& Richardson, 2012; Wagner et al., 2014; Webb et al., 2017). Метаанализ 12 компьютерных вмешательств на основе СВТ при депрессии выявил умеренный размер эффекта после лечения $(\mathrm{d}=0.56)$, отражающий большее улучшение симптомов по сравнению с участниками, включенными в контрольные группы (Webb et al., 2017). Размер этого эффекта уменьшался в зависимости от того, были ли эти программы с 
сопровождением участников $(\mathrm{d}=0.61)$ или без $(\mathrm{d}=0.25)$. В нашем исследовании мы получили высокий размер эффекта в уменьшении симптомов депрессии как при внутригрупповом сравнении $(\mathrm{d}=1.126)$, так и при межгрупповом сравнении с контрольной группой $(\eta 2=.175)$, что говорит о высоком потенциале программы по сравнению с другими решениями в данной области.

Учитывая, что тревожность, стресс и нарушения сна часто является проблемами коморбидными депрессии, важно отметить, что программа «Анти-депрессия» iCognito также существенно снижает симптомы тревоги, стресса и нарушения сна, что потенциально представляет программу как более широкий инструмент. Стоит отметить, что вмешательства, которые эффективны для уменьшения не только симптомов депрессии, но и различных сопутствующих коморбидных симптомов, могут быть очень полезны в клинической практике, учитывая большое количество сопутствующих заболеваний в данном состоянии (Fitzpatrick et al., 2014; Richardson \& Richardson, 2012).

Предыдущие исследования показывают, что вмешательства іСВТ увеличивают способность участников контролировать мысли, что можно рассматривать как предпосылку сопротивления депрессивным мыслям (Andersson et al., 2016; Baumeister et al., 2014). Наше исследование также подтверждает данный механизм изменений. За время участия в программе у испытуемых снизился уровень руминации и повысилась позитивная 
ориентация на решение проблемы. Таким образом, мы можем предполагать, что у участников начали формироваться новые когнитивные паттерны. Помимо этого, у участников экспериментальной группы был отмечен рост самоэффективности, осознанности и самосострадания, что дополнительно свидетельствует о важности данных элементов в терапии депрессии.

Наблюдаемый процент выбытия вмешательства в 65\% сопоставим с показателем метаанализа неуправляемых веб-вмешательств при депрессии, в котором в среднем около 40\% участников группы вмешательства выбыли, не завершив 25\% модулей (Karyotaki et al., 2015). Действительно, мета-анализ 40 исследований іСВТ показал, что более половины (57\%) пациентов, которым назначено лечение, выбывают из программы. Однако важно, чтобы в последующих испытаниях были приняты условия для повышения приверженности исследованному вмешательству іСВТ, чтобы соблюсти ограничение, заключающееся в том, что, несмотря на высокую приемлемость, процент выбытия вмешательства был высоким, в основном из-за недостатка времени или мотивации. Это может быть достигнуто путем оптимизации вмешательства (например, с помощью напоминаний или предоставления участникам возможности выбирать последовательность модулей), или путем изучения вмешательства в рамках комбинированного лечения (совместно с очной терапией). 
Стоит подчеркнуть, что большинство участников высоко оценили комфорт общения и надежность чат-бота. Это согласуется с результатами исследований, предполагающих, что терапевтические отношения могут быть установлены между людьми и искусственным интеллектом в контексте психического здоровья (Bickmore, Shulman, \& Yin, 2010). Предполагается, что взаимодействие с чат-ботом может «приблизить» терапевтический процесс и что изучение взаимозаменяемости и взаимодополняемости человека и искусственного интеллекта в этой области предлагает направление для будущих исследований.

\section{Ограничения}

В этом исследовании есть несколько методологических недостатков, которые ограничивают возможность обобщения результатов. Проведенное вмешательство было относительно коротким и не может отражать стабильность полученных эффектов во времени. Следовательно, исследование следует повторить с большим количеством участников и с более длительным периодом наблюдения, чтобы проверить, согласуются ли результаты. Более того, в выборке этого исследования почти не было мужчин; таким образом, влияние программы на них неизвестно. В целом мы получили небольшое количество заявлений от мужчин, и только один мужчина выполнил задания, чтобы иметь право на участие во втором опросе. Это согласуется с результатами национального опроса, согласно 
которому мужчины в России обращаются за профессиональной психологической помощью в четыре раза реже, чем женщины (Исследование фонда общественного мнения России, 2019).

Мы решили использовать подход на основе протокола в пилотном исследовании, чтобы определить возможный эффект лечения в «оптимальных условиях» и определить возможность дальнейших исследований (Gupta 2011; Feinman, 2009). Мы считаем, что будущее исследование эффективности на большей выборке следует проводить на основе анализа намерения лечить (ITT), чтобы учесть последствия несоблюдения режима лечения и оценить эффективность программы в условиях, более близких к реальным (Ranganathan et al., 2016).

\section{Литература}

1. Andersson G, Wagner B, Cuijpers P. ICBT for Depression. In: Lindefors $\mathrm{N}$, Andersson G, editors. Guided Internet-Based Treatments in Psychiatry. Springer International Publishing; 2016. pp. 17-32.

2. Andrews G, Cuijpers P, Craske MG, et al.: Computer therapy for the anxiety and depressive disorders is effective, acceptable and practical health care: a meta-analysis. PLoS One 2010; 5:e13196

3. Arkowitz H, Burke BL. Motivational interviewing as an integrative framework for the treatment of depression / Chapter in Motivational 
interviewing in the treatment of psychological problems, New York Guilford Press, 2008.

4. Baumeister H, Reichler L, Munzinger M, Lin J. The impact of guidance on Internet-based mental health interventions - A systematic review. Internet Interv. 2014;1(4):205-215.

5. Beck A., Rush J. et al. Cognitive Therapy of Depression, The Guilford Press, 1987.

6. Berger T, Krieger T, Sude K, Meyer B, Maercker A. Evaluating an emental health program ("deprexis") as adjunctive treatment tool in psychotherapy for depression: Results of a pragmatic randomized controlled trial. J Affect Disord 2018 Feb; 227:455-462.

7. Bickmore, T., Gruber, A., \& Picard, R. (2005). Establishing the computer-patient working alliance in automated health behavior change interventions. Patient Educ Couns, 59(1), 21-30.

8. Burns D. Feeling Good: The New Moods Therapy, Harper 2012.

9. Caldeira C, Chen Y, Chan L, Pham V, Chen Y, Zheng K. Mobile apps for mood tracking: an analysis of features and user reviews. AMIA Annu Symp Proc. 2018 Apr 16;2017:495-504.

10.Carlbring P, Andersson G, Cuijpers P, Riper H, Hedman-Lagerlöf E. Internet-based vs face-to-face cognitive behavior therapy for psychiatric and somatic disorders: an updated systematic review and meta-analysis. Cogn Behav Ther 2018 Jan; 47(1):1-18. 
11.Cook, S., Kudryavtsev, A.V., Bobrova, N. et al. Prevalence of symptoms, ever having received a diagnosis and treatment of depression and anxiety, and associations with health service use amongst the general population in two Russian cities. BMC Psychiatry 20, 537 (2020).

12.Darcy A, Daniels J, Salinger D, Wicks P,Robinson A Evidence of Human-Level Bonds Established With a Digital Conversational Agent: Cross-sectional, Retrospective Observational Study. JMIR Form Res 2021;5(5):e27868

13.Eells TD, Barrett MS, Wright JH, et al.: Computer-assisted cognitivebehavior therapy for depression. Psychotherapy 2014; 51:191-197

14.Ellis A. Overcoming Destructive Beliefs, Feelings and Behaviours: New Directions for Rational Emotive Behavioral Therapy, Prometheus Books, 2001.

15.Eysenbach G. (2005) The Law of Attrition. J Med Internet Res 2005; 7(1): e11

16.Feinman R. D. (2009). Intention-to-treat. What is the question?. Nutrition \& metabolism, 6, 1 .

17.Firth J, Torous J, Nicholas J, Carney R, Pratap A, Rosenbaum S, Sarris J. The efficacy of smartphone-based mental health interventions for depressive symptoms: a meta-analysis of randomized controlled trials. World Psychiatry. 2017 Oct;16(3):287-98. 
18.Fitzpatrick KK, Darcy A, Vierhile M. Delivering Cognitive Behavior Therapy to Young Adults With Symptoms of Depression and Anxiety Using a Fully Automated Conversational Agent (Woebot): A Randomized Controlled Trial. JMIR Ment Health 2017 Jun 06;4(2):e19

19.Fleming T, Bavin L, Lucassen M, Stasiak K, Hopkins S, Merry S. Beyond the trial: systematic review of real-world uptake and engagement with digital self-help interventions for depression, low mood, or anxiety. J Med Internet Res. 2018 Jun 6;20(6):e199.

20.Foroushani PS, Schneider J, Assareh N: Meta-review of the effectiveness of computerised CBT in treating depression. BMC Psychiatry 2011; $11: 13110.1186 / 1471-244 X-11-131$

21.Gaffney H, Mansell W, Tai S. Conversational Agents in the Treatment of Mental Health Problems: Mixed-Method Systematic Review. JMIR Ment Health. 2019 Oct 18;6(10):e14166.

22.Guilbert P. The Compassionate Mind (Compassion Focused Therapy), Robinson, 2009.

23.Gupta S. K. (2011). Intention-to-treat concept: A review. Perspectives in clinical research, 2(3), pp.109-112.

24.Huguet A, Rao S, McGrath PJ, Wozney L, Wheaton M, Conrod J, Rozario S. A Systematic Review of Cognitive Behavioral Therapy and Behavioral Activation Apps for Depression. PLoS One. 2016 May 2;11(5):e0154248. 
25.Inkster B, Sarda S, Subramanian V. An empathy-driven, conversational artificial intelligence agent (Wysa) for digital mental well-being: realworld data evaluation mixed-methods study. J Mhealth Uhealth. 2018 Nov 23;6(11):e12106.

26.Jenkins, R., Lancashire, S., McDaid D. et al. (2007) Mental health reform in the Russian Federation: an integrated approach to achieve social inclusion and recovery. Bulletin of the World Health Organization, 85 (11), 821-900

27.Kanter J., Busch A. and L. Rusch, Behavioral Activation: Distinctive Features, Routledge, 2009.

28. Karyotaki E, Kleiboer A, Smit F, et al. Predictors of treatment dropout in self-guided web-based interventions for depression: an "individual patient data" meta-analysis. Psychol Med. 2015;45(13):2717-2726.

29. Kirschenbaum H. Values Clarification in Counseling and Psychotherapy: Practical Strategies for Individual and Group Settings, Oxford Scholarship Online, May 2013.

30.Krasnov, V.N., Gurovich, I. (2012). History and current condition of Russian psychiatry. International Review of Psychiatry, 24 (4), 328-333.

31.Lackner R., Moore M. et al. Explanatory flexibility and explanatory style in treatment- seeking clients with Axis I psychopathology, Cognitive Therapy and Research, December 2015; 1-39(6).

32.Linardon, J., Fuller-Tyszkiewicz, M. (2020) Attrition and adherence in 
smartphone-delivered interventions for mental health problems: a systematic and meta-analytic review, Journal of consulting and clinical psychology, vol. 88, no. 1, pp. 1-13

33. Marshall JM, Dunstan DA, Bartik W. The digital psychiatrist: in search of evidence-based apps for anxiety and depression. Front Psychiatry. 2019;10:831.

34.Marshall, J. M., Dunstan, D. A., \& Bartik, W. (2020). Effectiveness of Using Mental Health Mobile Apps as Digital Antidepressants for Reducing Anxiety and Depression: Protocol for a Multiple Baseline Across-Individuals Design. JMIR research protocols, 9(7), e17159

35.McManus S, Bebbington P, Jenkins R, Brugha T. (eds.) (2016) Mental health and wellbeing in England: Adult Psychiatric Morbidity Survey 2014.

36.McCarter, T. Depression Overview, American Health \& Drug Benefits, April 2008

37.Mezu A., Mezu C. Problem-Solving Therapy: A Treatment Manual, Springer Publishing Company, 2012; Markowitz JC, Weissman MM. Interpersonal psychotherapy: principles and applications. World Psychiatry. 2004; 3(3):136-139.

38.Muñoz RF. Using evidence-based internet interventions to reduce health disparities worldwide. J Med Internet Res. 2010 Dec 17;12(5):e60. 
39.Nersessova, K. S., Jurcik, T., \& Hulsey, T. L. (2019). Differences in beliefs and attitudes toward Depression and Schizophrenia in Russia and the United States. International Journal of Social Psychiatry, 65(5), 388398.

40.Pereira, J., Díaz, Ó. (2019) Using Health Chatbots for Behavior Change: A Mapping Study. Journal of Medical Systems 43, 135.

41.Qu C, Sas C, Daudén Roquet C, Doherty G. Functionality of Top-Rated Mobile Apps for Depression: Systematic Search and Evaluation. JMIR Ment Health 2020;7(1):e15321

42.Ranganathan, P., Pramesh, C. S., \& Aggarwal, R. (2016). Common pitfalls in statistical analysis: Intention-to-treat versus per-protocol analysis. Perspectives in clinical research, 7(3), 144- 146.

43. Richards D, Richardson T: Computer-based psychological treatments for depression: a systematic review and meta-analysis. Clin Psychol Rev 2012; 32:329-34210.1016/j.cpr.2012.02.004

44.Russian Public Opinion Fund Survey. Psichologicheskaya podderzhka I psihologi [Psychological Support And Psychologists] (2019, March 18) https://fom.ru/Obraz-zhizni/14183 (in Russian)

45.Segal Z. Mindfulness-Based Cognitive Therapy for Depression, Second Edition, The Guilford Press, 2012. 
46. So M, Yamaguchi S, Hashimoto S, et al.: Is computerised CBT really helpful for adult depression? A meta-analytic re-evaluation of CCBT for adult depression in terms of clinical implementation and methodological validity. BMC Psychiatry 2013; 13:11310.1186/1471-244X-13-113

47. Stawarz K, Preist C, Tallon D, Wiles N,Coyle D User Experience of Cognitive Behavioral Therapy Apps for Depression: An Analysis of App Functionality and User Reviews J Med Internet Res 2018;20(6):e10120

48.Thase ME, Wright JH, Eells TD, et al.: Improving the efficiency of psychotherapy for depression: computer-assisted versus standard CBT. Am J Psychiatry 2018; 175:24225010.1176/appi.ajp.2017.17010089

49.Torous, J., Nicholas, J., Larsen, M. E., Firth, J. \& Christensen, H. Clinical review of user engagement with mental health smartphone apps: evidence, theory and improvements. Evid. Based Ment. Health 21, 116119 (2018).

50.Torre Arias de la -, Jorge Gemma Vilagut, Amy Ronaldson, Antoni Serrano-Blanco, Vicente Martín, Michele Peters, Jose M Valderas, Alex Dregan, Jordi Alonso (2021) Prevalence and variability of current depressive disorder in 27 European countries: a population-based study Lancet Public Health 2021, May 4, 2021 
51.U.S. Substance Abuse and Mental Health Services Administration. (2018). Key substance use and mental health indicators in the United States: Results from the 2017 National Survey on Drug Use and Health (HHS Publication No. SMA 18-5068, NSDUH Series H-53). Rockville, MD: Center for Behavioral Health Statistics and Quality, Substance Abuse and Mental Health Services Administration. Retrieved from https://www.samhsa.gov/data/

52.Wagner B, Horn AB, Maercker A. Internet-based versus face-to-face cognitive-behavioral intervention for depression: A randomized controlled non-inferiority trial. J Affect Disord. 2014;152-154:113-121. doi: 10.1016/j.jad.2013.06.032.

53. Watkins E. Rumination-Focused Cognitive-Behavioural Therapy for Depression, The Guilford Press, 2016.

54.Webb, C. A., Rosso, I. M., \& Rauch, S. L. (2017). Internet-Based Cognitive-Behavioral Therapy for Depression: Current Progress and Future Directions. Harvard review of psychiatry, 25(3), 114-122.

55.Winkler, P. et al. (2017) A blind spot on the global mental health map: a scoping review of 25 years' development of mental health care for people with severe mental illnesses in central and eastern Europe, The Lancet Psychiatry, 4 (8), $634-642$.

56. World Health Organization database: Crude Suicide Mortality (per 100,000 population), accessed on 11.07.2021 
https://www.who.int/data/gho/data/themes/mortality-and-global-healthestimates\#

57.Wright JH, Owen JJ, Eells TD, et al.: Computer-assisted cognitivebehavior therapy for depression: a systematic review and meta-analysis. J Clin Psychiatry 2019

58.Wright J. H. and Matthew Mishkind, Computer-Assisted CBT and Mobile Apps for Depression: Assessment and Integration Into Clinical Care, FOCUS 2020 18:2, 162-168

59.Wu, A., Scult, M.A., Barnes, E.D. et al. Smartphone apps for depression and anxiety: a systematic review and meta-analysis of techniques to increase engagement. npj Digit. Med. 4, 20 (2021)

\section{Депрессия ЭГ}

\begin{tabular}{|c|c|c|c|c|c|c|c|}
\hline \multirow{2}{*}{ Показатель } & \multicolumn{2}{|c|}{ ДО } & \multicolumn{2}{|c|}{ ПОСЛЕ } & \multicolumn{1}{c|}{ - } & Уровень & Размер \\
\cline { 2 - 5 } & M & SD & M & SD & Стьюден & значимос & эффек \\
та & т & та d \\
\hline PSS & 27,6 & 5,03 & 22,5 & 6,78 & $5,048^{* * *}$ & 0,001 & 0,854 \\
& 9 & & 1 & & & & \\
\hline GAD & 12,6 & 3,84 & 7,54 & 3,70 & $6,463 * * *$ & 0,001 & 1,093 \\
& 3 & & & & & & \\
\hline
\end{tabular}




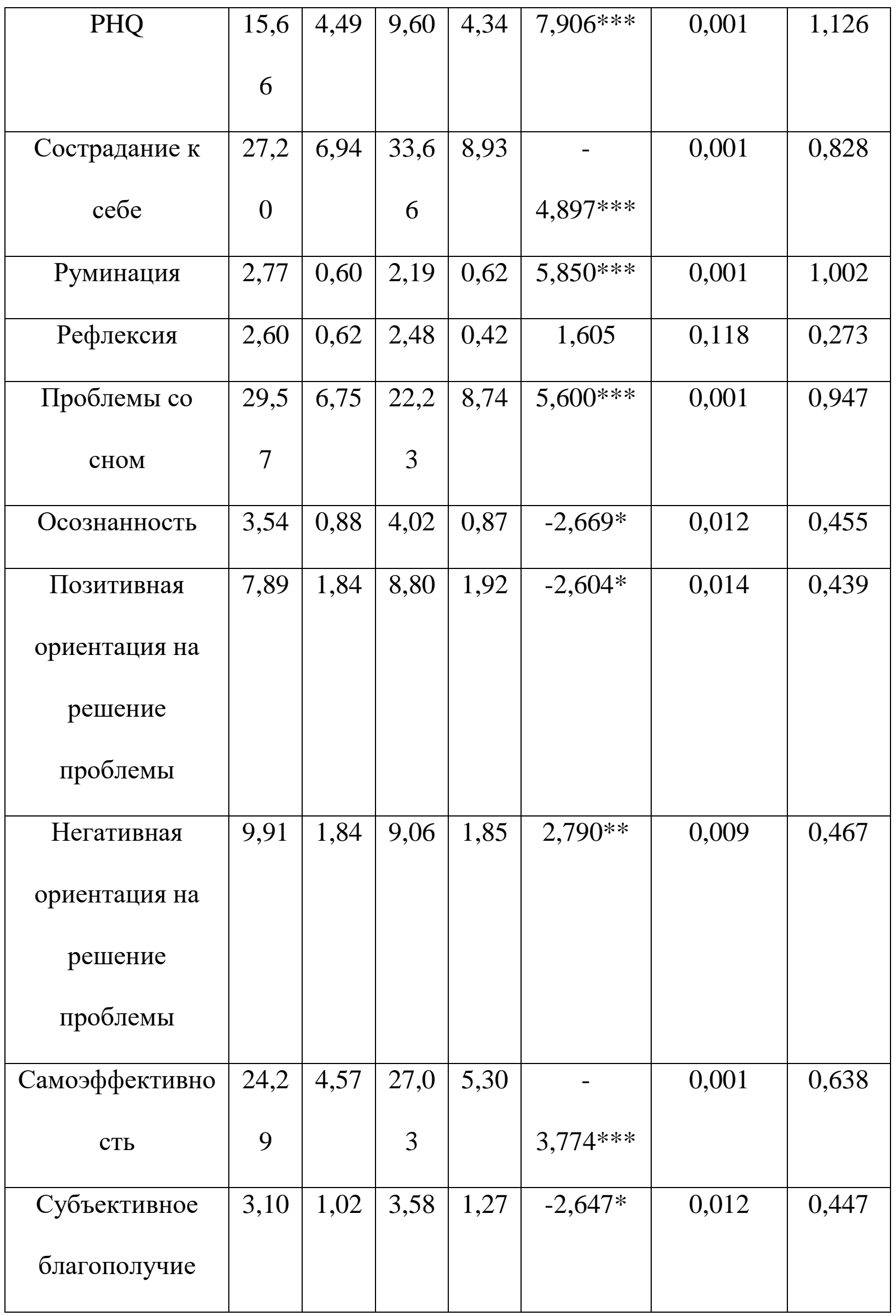




\begin{tabular}{|c|c|c|c|c|c|c|c|}
\hline Субъективное & 54,1 & 13,1 & 69,9 & 16,5 & - & 0,001 & 1,024 \\
благополучие 2 & 7 & 3 & 4 & 6 & $6,059 * * *$ & & \\
\hline Оптимизм & 31,7 & 9,45 & 38,0 & 10,7 & - & 0,001 & 0,691 \\
& 1 & & 3 & 1 & $4,083 * * *$ & & \\
\hline Конструктивная & 28,1 & 7,94 & 29,7 & 8,91 & $-1,788$ & 0,083 & 0,303 \\
коммуникация & 1 & & 7 & & & & \\
\hline
\end{tabular}

$\mathrm{N}=35$

32 женщины, 3 мужчины

Возраст $\mathrm{M}=31,37, \mathrm{SD}=9,80$

Средняя оценка Содержание $\mathrm{M}=4,27$

Дизайн $\mathrm{M}=4,74$

Удобство $\mathrm{M}=4,63$

Техники $\mathrm{M}=4,57$

Депрессия КГ (новая)

\begin{tabular}{|c|c|c|c|c|c|c|c|c|}
\hline Показатель & \multicolumn{2}{|c|}{ ДО } & \multicolumn{2}{|c|}{ ПОСЛЕ } & \multicolumn{1}{|c|}{$\mathrm{t}-$} & Уровень & & Разме \\
& $\mathrm{M}$ & $\mathrm{SD}$ & $\mathrm{M}$ & $\mathrm{SD}$ & Стьюде & значимо & & $\mathrm{p}$ \\
\hline
\end{tabular}




\begin{tabular}{|c|c|c|c|c|c|c|c|c|}
\hline & & & & & нта & сти $p$ & & $\begin{array}{c}\text { эффек } \\
\text { та d }\end{array}$ \\
\hline PSS & $\begin{array}{c}28,0 \\
7\end{array}$ & 4,20 & $\begin{array}{c}25,7 \\
6\end{array}$ & 5,19 & $2,978 * *$ & 0,005 & $\begin{array}{c}0,49 \\
6\end{array}$ & 0,484 \\
\hline GAD & $\begin{array}{c}12,1 \\
8\end{array}$ & 3,99 & $\begin{array}{c}10,4 \\
7\end{array}$ & 4,51 & $2,428^{*}$ & 0,020 & $\begin{array}{c}0,48 \\
5\end{array}$ & 0,392 \\
\hline PHQ & $\begin{array}{c}15,7 \\
3\end{array}$ & 4,25 & $\begin{array}{c}14,1 \\
6\end{array}$ & 5,06 & $2,183^{*}$ & 0,035 & $\begin{array}{c}0,55 \\
4\end{array}$ & 0,351 \\
\hline $\begin{array}{c}\text { Сострадание к } \\
\text { себе }\end{array}$ & $\begin{array}{c}27,2 \\
8\end{array}$ & 8,74 & $\begin{array}{c}28,1 \\
6\end{array}$ & 9,12 & $-0,998$ & 0,325 & & 0,104 \\
\hline Руминация & 2,67 & 0,69 & 2,71 & 0,65 & $-0,351$ & 0,728 & $\begin{array}{c}0,53 \\
9\end{array}$ & 0,062 \\
\hline Рефлексия & 2,49 & 0,67 & 2,43 & 0,65 & 0,619 & 0,540 & $\begin{array}{c}0,61 \\
7\end{array}$ & 0,103 \\
\hline $\begin{array}{c}\text { Проблемы со } \\
\text { сном }\end{array}$ & $\begin{array}{c}28,5 \\
0\end{array}$ & 7,30 & $\begin{array}{c}27,5 \\
2\end{array}$ & 6,54 & 0,928 & 0,359 & $\begin{array}{c}0,56 \\
9\end{array}$ & 0,152 \\
\hline Осознанность & 3,49 & 0,83 & 3,46 & 0,74 & 0,361 & 0,720 & $\begin{array}{c}0,68 \\
3\end{array}$ & 0,047 \\
\hline $\begin{array}{c}\text { Позитивная } \\
\text { ориентация на } \\
\text { решение }\end{array}$ & 7,42 & 1,88 & 7,81 & 1,86 & $-1,487$ & 0,145 & $\begin{array}{c}0,82 \\
0\end{array}$ & 0,348 \\
\hline
\end{tabular}




\begin{tabular}{|c|c|c|c|c|c|c|c|c|}
\hline проблемы & & & & & & & & \\
\hline Негативная & 10,2 & 1,50 & 9,76 & 1,73 & 1,825 & 0,076 & 0,61 & 0,351 \\
решентация на & 6 & & & & & & 7 & \\
проблемы & & & & & & & & \\
\hline Самоэффектив & 23,4 & 6,14 & 24,3 & 6,32 & $-1,645$ & 0,108 & 0,84 & 0,269 \\
ность & 7 & & 9 & & & & 7 & \\
\hline Субъективное & 2,95 & 1,16 & 3,04 & 1,34 & $-0,523$ & 0,604 & 0,65 & 0,095 \\
благополучие & & & & & & & 5 & \\
\hline Субъективное & 54,2 & 12,4 & 57,7 & 13,9 & $-2,471 *$ & 0,018 & 0,79 & 0,401 \\
\hline благополучие 2 & 9 & 5 & 6 & 3 & & & 0 & \\
\hline Оптимизм & 31,6 & 9,36 & 33,8 & 12,1 & $-1,616$ & 0,115 & 0,71 & 0,262 \\
\hline коммуникация & 5 & & 9 & 1 & & & 3 & \\
\hline Конструктивна & 30,7 & 7,79 & 31,4 & 8,10 & $-0,620$ & 0,539 & 0,60 & 0,100 \\
\hline
\end{tabular}

$\mathrm{N}=38$

Bсе женщины

Возраст $\mathrm{M}=24,89, \mathrm{SD}=5,15$ 
Межгрупповой эффект 2x2 ANOVA для связанных выборок

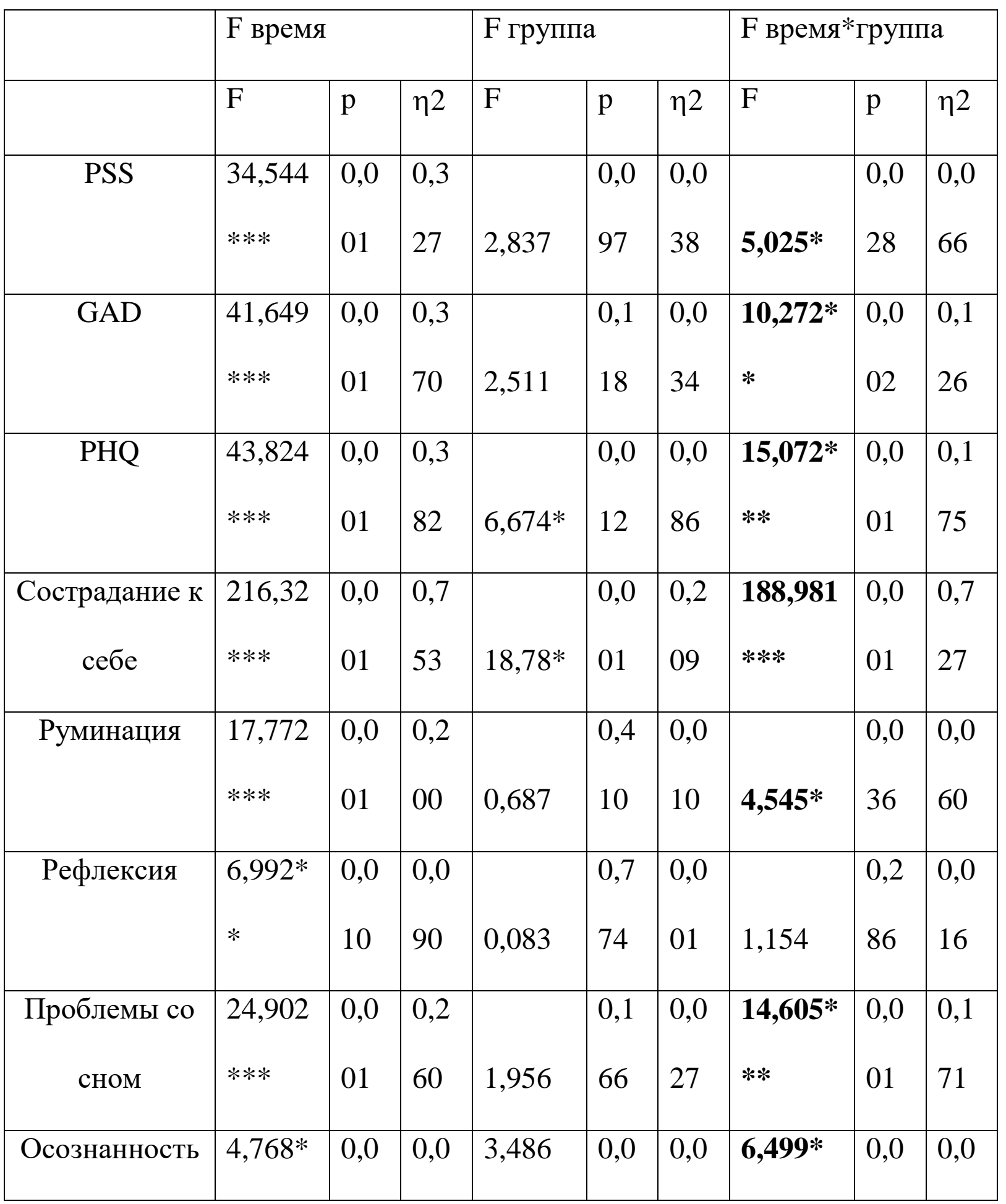




\begin{tabular}{|c|c|c|c|c|c|c|c|c|c|}
\hline & & 32 & 63 & & 66 & 47 & & 13 & 84 \\
\hline \multicolumn{10}{|l|}{ Позитивная } \\
\hline \multicolumn{10}{|l|}{ ориентация на } \\
\hline решение & 18,708 & 0,0 & 0,2 & 14,845 & 0,0 & 0,1 & $8,504 * *$ & 0,0 & 0,1 \\
\hline проблемы & $* * *$ & 01 & 09 & $* * *$ & 01 & 73 & $*$ & 05 & 07 \\
\hline \multicolumn{10}{|l|}{ Негативная } \\
\hline \multicolumn{10}{|l|}{ ориентация на } \\
\hline решение & & 0,6 & 0,0 & 13,488 & 0,0 & 0,1 & & 0,1 & 0,0 \\
\hline проблемы & 0,182 & 71 & 03 & $* * *$ & 01 & 60 & 1,765 & 88 & 24 \\
\hline Самоэффекти & 16,237 & 0,0 & 0,1 & & 0,1 & 0,0 & & 0,0 & 0,0 \\
\hline вность & $* * *$ & 01 & 86 & 1,914 & 71 & 26 & $4,014 *$ & 49 & 54 \\
\hline Субъективное & & 0,0 & 0,0 & & 0,1 & 0,0 & & 0,1 & 0,0 \\
\hline благополучие & $5,229 *$ & 25 & 69 & 1,788 & 85 & 25 & 2,459 & 21 & 33 \\
\hline \multicolumn{10}{|l|}{ Субъективное } \\
\hline благополучие & 44,199 & 0,0 & 0,3 & & 0,0 & 0,0 & $18,048 *$ & 0,0 & 0,2 \\
\hline 2 & $* * *$ & 01 & 84 & $4,148 *$ & 45 & 55 & $* *$ & 01 & 03 \\
\hline \multirow[t]{2}{*}{ Оптимизм } & 17,078 & 0,0 & 0,1 & & 0,3 & 0,0 & & 0,0 & 0,0 \\
\hline & $* * *$ & 01 & 94 & 0,884 & 50 & 12 & $3,883 *$ & 53 & 52 \\
\hline \multicolumn{10}{|l|}{ Конструктивн } \\
\hline ая & & 0,1 & 0,0 & & 0,2 & 0,0 & & 0,5 & 0,0 \\
\hline коммуникаци & 2,527 & 16 & 34 & 1,515 & 23 & 21 & 0,404 & 27 & 06 \\
\hline
\end{tabular}




\begin{tabular}{|l|l|l|l|l|l|l|l|l|l|}
\hline Я & & & & & & & & & \\
\hline
\end{tabular}

Как интерпретировать размер эффекта (ๆ2) для факторного анализа: $\eta^{2}=$ 0.01 indicates a small effect; $\eta^{2}=0.06$ indicates a medium effect; $\eta^{2}=0.14$ indicates a large effect. 